\title{
RELACIÓN ENTRE EL DESARROLLO VOCACIONAL EFICAZ Y LOS FACTORES DE ELECCIÓN EN TITULADOS UNIVERSITARIOS CON DISCAPACIDAD ACTIVOS LABORALMENTE. UN ESTUDIO PRELIMINAR
}

\section{RELATIONSHIP BETWEEN CAREER DEVELOPMENT AND THE FACTORS OF CAREER CHOICE IN UNIVERSITY ASSETS OCCUPATIONALLY DISABLED. A PILOT STUDY}

\author{
María Ángeles Segura García \\ José Manuel Martínez-Vicente ${ }^{1}$ \\ Isabel García Martínez \\ Universidad de Almería. \\ Facultad de Psicología. \\ Almería. España
}

\section{RESUMEN}

Existe escasa evidencia sobre el desarrollo vocacional de estudiantes universitarios con discapacidad y menos aún cuando éstos se encuentran insertados en el ámbito laboral. El objetivo de este estudio, fue comprobar la relación existente entre el desarrollo vocacional eficaz y los factores de elección vocacional en titulados universitarios con discapacidad insertados laboralmente. Para ello se empleó una muestra de 30 sujetos de una edad media de 38.23 años

\footnotetext{
${ }^{1}$ Correspondencia: José Manuel Martínez Vicente: Ctra. De Sacramento s/n. La cañada de San Urbano. 04120-Almería. Correo-e: jicente@ual.es
} 
$(\mathrm{Sd}=9.2)$ a los que se le aplicó un cuestionario de biodatos universitarios y un Inventario de factores de elección vocacional. Se empleó un diseño correlacional e inferencial realizándose análisis de correlaciones, de regresión y la pruebas de Krusal-Wallis y U de Mann-Whitne en los análisis inferenciales Los resultados obtenidos corroboran, que el desarrollo vocacional eficaz quedó asociado, a los gustos y preferencias en las elecciones que realizaron. También se apreció, que a mayor desarrollo vocacional eficaz, menos influencia ejercieron los factores de carácter sociogénico y su propia discapacidad en la elección vocacional. Se concluye, que el conocimiento del desarrollo vocacional de estos sujetos, puede repercutir en mejorar los procesos de asesoramiento vocacional y profesional de este colectivo.

Palabras clave: discapacidad, conducta vocacional, factores de elección vocacional, desarrollo vocacional, titulados universitarios

\section{ABSTRACT}

There is little evidence about vocational development of college students with disabilities and even less when they were inserted in the workplace. The objective was to determine the correlation between the vocational development and career choice factors of graduates with disabilities inserted occupationally. For this was used 30 university subjects with an average age of 38.23 years $(\mathrm{Sd}=9.2)$ with various disabilities inserted occupationally to which was applied a questionnaire university biodata and an inventory of vocational factors, to a sample. A correlational design was used and inferential performing correlation analysis, and regression testing using Kruskal-Wallis and Mann-Whitne in inferential analysis. The results obtained confirm that the effective vocational development was associated with the tastes and preferences in the choices they made. Was also noted that the higher effective vocational development, exert less influence factors sociogenic character and their own disability. We conclude that knowledge of career development of these subjects may influence improving the processes of vocational and professional advice of this group.

Keywords: Disability, vocational behaviour, career choice factors, vocational development, university graduates.

\section{Introducción}

Según los datos de la comisión sobre la igualdad y la no discriminación del consejo de Europa (2014), existen más de 80 millones de personas afectadas por alguna discapacidad en la Unión Europea. En el caso de España, alrededor de 2,5 millones con discapacidad reconocida.

Ante esta situación, surge la necesidad de crear estrategias tanto internacionales como nacionales, que consigan mejorar la igual de derechos en todos los ámbitos de la vida social y laboral. La estrategia llevada a cabo por la unión europea sobre discapacidad (2010-2020), subraya la necesidad, de acceso a una educación de calidad y aprendizaje permanente. Con el objetivo de que las personas con discapacidad, obtengan una mejora en su calidad de vida y una plena participación en la sociedad. La Comisión europea ha identificado ocho ámbitos fundamentales de actuación: accesibilidad, participación, igualdad, empleo, educación y formación, protección social, sanidad y acción exterior. Determinando medidas clave para cada uno de ellos, por ejemplo, en el ámbito empleo: Prestar especial atención a los jóvenes con discapacidad, en su transición de la educación al empleo. 
En el ámbito nacional y siguiendo la directrices europeas, se esta llevando a cabo la estrategia española sobre discapacidad (2014-2020), con el objetivo de alcanzar las medidas establecidas en la estrategia europea 2020.

Centrándonos en el ámbito universitario, es destacable el incremento de estudiantes con discapacidad que han accedido a titulaciones superiores en los últimos años, como se puede comprobar en los datos publicados en el II Estudio de Universidad y Discapacidad, llevado a cabo por la Fundación Universia (2014), donde se da a conocer el estado actual de inclusión, de la personas con discapacidad en el sistema universitario español, confirma que el número de estudiantes con discapacidad ha aumentado de un 1,1\% en el curso académico 2011/2012 a un $1,3 \%$ en el curso $2013 / 2014$.

Este incremento, unido a la incorporación al Espacio Europeo de Educación Superior, junto a las normativas introducidas sobre discapacidad, 1) Ley Orgánica de universidades 4/2007, de 12 de abril, de Universidades, donde se "garantizarán la igualdad de oportunidades de los estudiantes y demás miembros de la comunidad universitaria con discapacidad..."; 2) Ley General de Discapacidad de 2013 y Real Decreto por el que se aprueba el Estatuto del Estudiante Universitario de 2010, han planteado la importancia de ofrecer programas y servicios específicos que puedan dar respuesta a las necesidades de cada alumno con discapacidad de forma individualizada. Así lo confirma, una investigación llevada a cabo por Rodríguez y Álvarez (2015), en estudiantes universitarios, estableciendo la necesidad de desarrollar tutorías especificas, en función de las necesidades de cada alumno, con especial atención a la integración de nuevos estudiantes. También aconsejan establecer programas de tutorías permanentes, para los estudiantes con discapacidad durante toda su formación. Con ello se introducen nuevos elementos que condicionan el desarrollo vocacional de esta población.

Partiendo de este punto, nuestro estudio nace de la necesidad de conocer de forma específica, a través de una muestra de titulados universitarios con discapacidad, la conducta que adoptó este tipo de población durante su formación y su posterior desarrollo profesional. Tomando como referencia dos constructos que modulan el desarrollo de la conducta vocacional: los biodatos y los factores de elección vocacional.

La conducta vocacional podemos entenderla ampliamente como una manifestación comportamental compuesta de pensamientos, acciones y actitudes, que el individuo despliega en su medio social y cultural, con la intención de adquirir experiencias y formación, que le lleven a establecer planes de acción para su futura incorporación en el mundo adulto y laboral y al desarrollo de su futura carrera profesional. Por su parte Rivas $(1988,2003)$ la define como el “ conjunto de procesos psicológicos que una persona concreta pone en marcha en relación con el proceso de acceso al mundo profesional adulto en el que pretende insertarse activamente, o en el que ya está insertado". Para este autor la discapacidad representa un codeterminante más de la conducta vocacional. En este sentido Ávila, Celeste y Rocabert (2003), de manera similar, la consideran como un codeterminante que no fija o determina una única vía de desarrollo personal, y que puede o no, orientarse al desarrollo profesional. Por otra parte estos autores consideran la normalización como el proceso que permitirá que la conducta vocacional de estas personas pueda alcanzar su culminación en el proceso de socialización. Podemos señalar que puede ser el factor más notorio de la conducta vocacional de estas personas. De hecho la toma de decisiones vocacionales puede variar en función del tipo y grado de discapacidad o volverse más compleja si el individuo no dispone de la información necesaria o depende de otros para poder desarrollar sus planes de futuro (Luque, 2006).

Polo y López-Justicia (2006) ponen de manifiesto algunos de los condicionantes o barreras que determinan el desarrollo vocacional de la persona discapacitada como son las deficiencias en la formación, la falta de experiencia, la excesiva protección familiar y los referentes erróneos sobre discapacidad. En un estudio piloto realizado con 45 estudiantes universitarios con discapacidad, 
White, Summers y Renault (2014) concluyen que estos estudiantes se encuentran en mayor riesgo de experimentar dificultad en la gestión de los factores contextuales y ambientales relacionados con el proceso de desarrollo de la carrera en comparación con sus homólogos sin discapacidad. Por su parte, Ávila y Rocabert (2001), en un estudio realizado con deficientes visuales, constataron las barreras que afectan al desarrollo vocacional de estos estudiantes señalando que además de las barreras arquitectónicas, de infraestructuras, equipamientos, método empleado en clase y los materiales de estudio, existen otras menos explícitas relacionadas con la falta de un asesoramiento vocacional en periodos anteriores así como la falta de conocimiento sobre el mundo del trabajo. Otros autores, como Vila y Pallisera (2002), hacen mención al autoconcepto como otro tipo de condicionante que puede afectar en gran medida al desarrollo vocacional de las personas discapacitadas. De manera similar Valiante (2000) constata que las convicciones que una persona puede tener acerca de sus capacidades pueden ser un mejor predictor de la conducta posterior que su nivel de habilidad real. Por otro lado Cheathan, Smith, Elliot y Friedline (2013) señalan las dificultades económicas y las bajas expectativas académicas como obstáculos que las personas con discapacidad tienen de cara a los estudios universitarios. Así mismo destacan el papel de la familia para abordar este reto.

TABLA 1. Estudios realizados sobre los factores que influyen en el desarrollo vocacional de personas con discapacidad

\begin{tabular}{|c|c|}
\hline Autores & $\begin{array}{l}\text { Factores que influyen en el desarrollo vocacional de } \\
\text { las personas con discapacidad }\end{array}$ \\
\hline Valiante (2000) & Convicciones sobre su capacidad. \\
\hline Ávila y Rocabert (2001) & $\begin{array}{l}\text { Sobreprotección familiar, metodología utilizada en el } \\
\text { aula, barreras arquitectónicas e infraestructuras, } \\
\text { materiales de estudio, desconocimiento mundo laboral. }\end{array}$ \\
\hline Vila y Pallisera (2002) & Autoconcepto. \\
\hline Polo y López-Justicia (2006) & $\begin{array}{l}\text { Las deficiencias en la formación, la falta de experiencia, } \\
\text { la excesiva protección familiar y los referentes erróneos } \\
\text { sobre discapacidad. }\end{array}$ \\
\hline Cheathan, Smith, Elliot y Friedline (2013). & $\begin{array}{c}\text { Dificultades económicas y bajas expectativas } \\
\text { académicas. }\end{array}$ \\
\hline
\end{tabular}

Como señalan Martínez-Vicente, Segura y García (2014) existe escasa evidencia relacionada con las características vocacionales de los estudiantes universitarios con algún tipo de discapacidad y en menor medida si estos se encuentran insertados laboralmente. Sin embargo si se cuenta con estudios que han tratado de caracterizar la conducta vocacional y preprofesional de estudiantes universitarios (Cabrera y Bethencourt, 2008; Gomez-Artiaga, Lloret, Rocabert, y Descals, 2013; Mora, 2008; Rivas, 2005; Rocabert y Autor, 2011).

El concepto de codeterminación, en relación a la conducta vocacional, definido por Rivas (2003) lleva implícito el papel que representa la influencia tanto de los factores de origen social (sociogénicos: condicionantes familiares, estereotipos que señalan poder y prestigio profesional y condicionantes escolares o académicos) y los factores de origen individual (psicogénicos: madurez vocacional y asesoramiento vocacional) en el desarrollo de la conducta vocacional. En este mismo sentido otros autores (Amir, Gati y Kleiman, 2008; González Maura, 2006; Uribarri, 2004) detallan también factores o condicionantes, tanto personales como sociales, que influyen en la conducta vocacional y recogen la relación existente entre ellos.

Por su parte, Pascual (2006) realiza un estudio donde aparece la oportunidad como un factor que afecta fundamentalmente a la toma de decisiones vocacionales. En este sentido una vez que operacionaliza la variable situación vocacional actual (ventajosa y no ventajosa), esta obtiene una correlación de $.38(p<.001)$ con la oportunidad de formación y de .26 $(p<.001)$ con la preparación 
para la oportunidad. Desde su punto de vista la oportunidad, trasciende al azar e implica capacidad del individuo para identificar, procesar y aprovechar ciertas circunstancias que se le puedan presentar y planificar su conducta con la intención de conseguir su meta vocacional.

Llegados a este punto, nuestra investigación tuvo en cuenta a la hora de evaluar los factores de elección vocacional, tanto factores de tipo sociogénico y psicógenico, como referidos a la oportunidad y la propia discapacidad como codeterminantes del desarrollo vocacional de estos sujetos. Pretendemos constatar cuáles de estos factores ejercieron mayor influencia en el desarrollo vocacional de los sujetos con discapacidad.

Otro de los constructos objeto de este estudio fueron los biodatos. Para Rocabert, Descals y Gómez (2007) los biodatos son el referente experiencial que el individuo aporta a la conducta vocacional con el objeto de conseguir su integración sociolaboral. Estas autoras señalan además que el contenido de los biodatos puede encuadrarse en varias categorías: datos demográficos, académicos, familiares, tiempo de ocio, relaciones sociales, etc. De todos ellos son los biodatos académicos los que demuestran una mayor relación con los intereses y la toma de decisiones con respecto a un oficio o carrera profesional (Dosil y Fernández Eire, 2001; Gimeno, 2003; Martín, 2006).

Por todo ello creemos necesario, a la hora de analizar la conducta vocacional de los universitarios, explorar aquellos biodatos que puedan informarnos de las vivencias, hechos y conductas del estudiante en relación a su desarrollo vocacional para comprobar la adecuación o eficacia de las mismas a la hora de planificar y explorar sus opciones de especialización y preparación de su profesionalidad. Estudios como los realizados por Rocabert, Descals y Gómez (2007), constatan que la historia personal de los sujetos y sus experiencias vividas, aportan al sujeto un determinado valor en su conducta vocacional. Por su parte, los biodatos académicos han tenido siempre una importancia relevante a la hora de estudiar la conducta vocacional, ya que el nivel educativo de los individuos, ha sido un codeterminante de primer orden para el acceso al mundo laboral. Los Itinerarios formativos, a su vez, han reflejado la formación escolar individual lograda a través de la opcionalidad de las asignaturas seguidas en los currículos escolares, tanto en secundaria como en estudios superiores.

Para Martínez-Vicente y Rocabert (2014) los biodatos representan un indicador del desarrollo vocacional del sujeto. Estos autores comprueban mediante un análisis factorial la estructura del constructo, verificando que los factores obtenidos de primer orden confluyen en un solo factor que denominan desarrollo vocacional eficaz. En este sentido los biodatos evaluados responden a una estructura factorial configurada por tres factores: la satisfacción de la elección, la preparación para el futuro profesional y las dificultades para la toma de decisiones. Un análisis factorial de segundo orden les permitió aglutinar a los tres citados factores anteriores en un solo factor, que los autores lo consideran como un indicador de desarrollo vocacional. Un estudios previo de Rocabert y Martínez-Vicente (2011) verifica la capacidad de este constructo para obtener patrones diferenciales en relación con la preparación para la carrera profesional, la personalidad eficiente y la identidad vocacional.

Un desarrollo vocacional satisfactorio irá unido a un conjunto de características personales que tienen los sujetos de enfrentarse con éxito a su entorno. Una persona dotada con este perfil, sería aquella que presenta y utiliza algunas de las siguientes características: una alta asertividad, autoestima, capacidad de trabajo, confianza en sí mismo, estabilidad emocional, capacidad de aprender de la experiencia, iniciativa, motivación de logro, optimismo, perseverancia,... (Gati, Landman, Davidovitch, Asulin-Peretz y Gadassi, 2010; Martín del Buey, Martín, Fernández, Dapelo y Marcone, 2004; Mehran, 2010; Staudinger y Bowen, 2010).

La inexistencia de estudios específicos, referentes al desarrollo de la conducta vocacional y factores de elección de titulados universitarios con discapacidad insertados laboralmente hace que 
se plantee este estudio con la intención de obtener unos resultados que puedan ser extrapolados a futuros estudiantes con discapacidad que pretendan desarrollar estudios superiores, así como ser tenidos en cuenta en los procesos de asesoramiento, orientación e inserción laboral con el objetivo de mejorar las condiciones y la calidad de su desarrollo vocacional. En este sentido el objetivo general fue comprobar la relación existente entre el desarrollo vocacional y los factores de elección vocacional en titulados universitarios con discapacidad insertados laboralmente.

\section{Método}

\section{Muestra}

La muestra quedó formada por 30 sujetos con titulación universitaria insertados laboralmente, de los cuales $50 \%$ fueron hombres y otro $50 \%$ fueron mujeres con un rango de edad de 23 a 55 años, siendo la edad media de 38.23 años y una desviación típica de 9.198. Atendiendo al tipo de discapacidad la mayor parte de la muestra contaba con una discapacidad motora (43.3\%) seguida de discapacidad múltiple (33.3\%). La discapacidad visual y auditiva contó con el mismo porcentaje $(10 \%)$ y la discapacidad psíquica quedó representada con un 3.3\%. En relación con la profesión la mayor parte de la muestra contaba con titulaciones relacionadas con las Ciencias Sociales y Jurídicas (40\%) y con las Artes y las Humanidades (27\%) el resto se distribuyó entre las Ciencias (13\%), las Ciencias de la Salud y las Ingenierías y la Arquitectura con un 10\% respectivamente.

\section{Instrumentos}

\section{Cuestionario de Biodatos Universitarios (CBU).}

El cuestionario de biodatos supone una adaptación del Cuestionario de Biodatos Universitarios (CBU) de Rocabert y Martinez-Vicente (2011) a sujetos con discapacidad, en las que se han introducido opciones de respuesta relacionadas con la temática que nos ocupa

Se trata de un cuestionario de autoinforme que solicita al sujeto que seleccione aquella opción que mejor defina su situación en cada indicador, pudiendo, en alguna de ellas, escoger más de una alternativa. Sus autores tras aplicarlo a una muestra de 2164 estudiantes universitarios señalan la bondad psicométrica del mismo en relación a su homogeneidad y su capacidad de permitir clasificar a los sujetos en función de su Desarrollo de Carrera Eficaz. Hecho que corroboran con el buen comportamiento del cuestionario para discriminar a los estudiantes en función de otros ámbitos de la conducta pre-profesional a saber: identidad vocacional, estudio, psicoemocionalidad, preparación para la carrera profesional. Por otra parte Martínez-vicente y Rocabert (2014) obtienen a partir de un análisis factorial tres factores de primer orden:

- Interés y satisfacción con la elección realizada.

- Elecciones para preparar el futuro profesional.

- Toma de decisiones respecto a la especialidad o área de trabajo.

Tras realizar un análisis factorial de segundo orden obtuvieron un único factor al que denominaron Desarrollo Vocacional Eficaz, demostrando la validez del cuestionario para medir el citado constructo. 
El cuestionario de Biodatos empleado quedó configurado por 6 indicadores que abarcan diferentes biodatos académicos relacionados con las experiencias vividas y las conductas manifestadas por los sujetos en relación con su desarrollo vocacional:

- Lugar de la opción elegida después de finalizar la selectividad (1ํㅜㄴํํㄴ $3^{\circ}, 4^{\circ}$ opción)

- Periodos de edad en los que le interesaron los estudios cursados (12-14, 15-16, 17-18, más de 19 años)

- Por qué escogió la carrera cursada (por mis aptitudes, por mi discapacidad, la nota media para entrar, posibilidades económicas, encontrarse cerca de mi residencia, salidas profesionales, consejo familia, consejo orientador, conocía personas con discapacidad que la había cursado, por mis intereses preferencias vocacionales)

- Grado de satisfacción con la elección realizada

- Influencia de la discapacidad en la toma de decisiones vocacionales

- Dificultades percibidas en el proceso de toma de decisiones preprofesionales (ninguna opción me satisfacía plenamente; no tenía clara la opción profesional que más me convenía; me faltaba información fiable sobre mi mismo; que mis preferencias profesionales cambiasen en un futuro; desconocía que es importante tener en cuenta para decidir adecuadamente; no tenía contactos importantes que me ayudasen a conseguir el trabajo que quería; personas importantes para mi no apoyaban mis planes; no tenía dificultades personales en decisión; no tenía la información académico-profesional que hacía falta.

A cada una de las anteriores cuestiones, por acuerdo interjueces, se le asignó una puntuación que conjuntamente con el resto conformaron la puntuación de nivel de Desarrollo Vocacional. Más información al respecto se puede ver en el apartado de procedimiento

\section{Inventario de factores vocacionales de Rivas y Pascual (2003).}

Este inventario está configurado por 30 items que evalúan siete factores que configuran tres fuentes. La primera de ellas hace referencia a la influencia externa a la conducta vocacional (sociogénesis) que queda formada por los factores: condicionantes del medio familiar, estereotipos que señalan poder y prestigio profesional, condicionamientos escolares 0 académicos contextuales. Por otro lado, hay una parte de ítems que expresan las influencias de carácter individual, psicogénesis, que se materializan en términos de madurez y asesoramiento vocacional recibido. Además surge una tercera fuente a la que denominan oportunidad que se desdobla en circunstancias que la preparan y la formación.

Dada la población diana de estudio se introdujeron 4 nuevos items relacionados con el papel de la discapacidad en la elección. En consecuencia el inventario quedó configurado por 34 items con los que se pretendía obtener información de los siguientes factores:

- Condicionantes familiares: Influencia de los padres o algún familiar, ambiente de casa, presión familiar respecto a selectividad, experiencias familiares que mediatizan el desarrollo vocacional de los sujetos.

- Estereotipos sociales (Poder/Prestigio): Salidas profesionales, prestigio de los estudios, poder económico y social que se quería conseguir, ser una carrera que se ajustase a las condiciones de hombre o mujer. 
- Condicionantes académicos: Dificultad / facilidad / duración de los estudios, horario y tiempo disponible, estudiar en la proximidad de casa.

- Madurez vocacional: Expectativas de éxito en los estudios elegidos, percepción de control sobre los acontecimientos, gustos y preferencias, éxito en materias relacionas con la opción vocacional que eligió , autoconocimiento, conocimiento del mundo laboral al que se dirigía, constancia, capacidad de trabajo y sacrificio.

- Asesoramiento vocacional: Asesoramiento vocacional recibido, haber conocido a una persona relevante del área vocacional elegida, apoyo, ayuda, estimulo de algún profesor/a.

- Preparación para la oportunidad: Alerta para aprovechar en función del tiempo disponible y economía familiar, las posibilidades que se cruzan en el camino profesional, la preparación personal a lo largo de los años, aprovechando las oportunidades de formación que hayan ido presentando, la suerte.

- Oportunidad de formación /proyección: Alerta y aprovechamiento de las posibilidades de formación novedosas, en lo que respecta a sus salidas profesionales y a la confianza personal que tenía en desarrollar con éxito tales estudios, así como las profesiones que implica, la casualidad.

- Propia discapacidad: Influencia de la propia discapacidad en su elección vocacional, adaptabilidad del entorno, recursos materiales y humanos para su integración, barreras arquitectónicas que pudiesen influir.

Calculada su fiabilidad se obtuvo un coeficiente alpha de Cronbach de .91, con lo que consistencia interna del cuestionario fue adecuada.

\section{Procedimiento}

La muestra se obtuvo a través de Servicios de Apoyo de las universidades, asociaciones de personas con discapacidad (ONCE, Federación Andaluza de Asociaciones de personas con discapacidad,...) y distintas Fundaciones de distintas zonas geográficas de España, donde previamente habíamos solicitado su colaboración en el estudio. Se empleó un muestreo incidental donde no se seleccionaron los casos sino que se incluyeron todos aquellos sujetos que habían decidido participar en el estudio, dada la dificultad para conseguir muestra.

Una vez mostrado su interés por el estudio se contactó con las personas interesadas facilitándole información necesaria para cumplimentar los cuestionarios que se emplearon. Se comprobó de antemano que cumplían los requisitos establecidos para este estudio:

- Poseer titulación universitaria finalizada.

- Certificado de minusvalía del $33 \%$ o superior.

- Estar activo laboralmente.

Se le facilitó en todo momento la ayuda que fue demandada adaptándonos al tipo de discapacidad. Aunque los cuestionarios eran anónimos la asignación de un código nos permitió verificar que la persona que respondía era realmente un sujeto del estudio con el que se había 
contactado previamente. En otros casos se empleaba la comunicación telefónica para verificar la identidad de la cumplimentación de los cuestionarios.

La aplicación del protocolo de investigación se llevó a cabo de forma on-line (Google Docs).

Se empleó la puntuación total de biodatos como un indicador de desarrollo vocacional eficaz. Para ello se puntuaron las opciones de respuestas consideradas como predictoras de un desarrollo vocacional eficaz, para ello se tuvo en cuenta el criterio establecido por acuerdo interjueces desarrollado por los autores del cuestionario de Biodatos. La puntuación máxima que se podía obtener en Biodatos fue de 25 y empleando los percentiles 25 y 75 se clasificaron a los sujetos de la muestra en:

- Desarrollo vocacional bajo: Formarían parte de este grupo aquellos sujetos que tuvieran una puntuación entre 0 y 15 en Biodatos.

- Desarrollo vocacional medio: Formarían parte de este grupo aquellos sujetos que tuvieran una puntuación entre 16 y 18 en Biodatos.

- Desarrollo vocacional alto: Formarían parte de este grupo aquellos sujetos que tuvieran una puntuación entre 19 y 25 en Biodatos.

Se realizó un estudio de carácter descriptivo correlacional e inferencial. Para llevar a cabo nuestro análisis de datos se empleó el SPSS en su versión 21 con licencia institucional.

\section{Resultados}

\section{Análisis de asociación entre Biodatos y Factores de elección vocacional}

Con el fin de comprobar el grado de relación existente entre los factores de elección vocacional y el desarrollo vocacional, representado en nuestra caso por la puntuación total obtenida en Biodatos, se correlacionó cada uno de los ítems que formaba parte del cuestionario con la citada puntuación. De los 34 items solo 7 obtuvieron una correlación estadísticamente significativa. En este sentido el item que presentó la correlación más elevada y de carácter positivo ( $r=.55, p>.01$ ) fue el 17 (Mis gustos y preferencias). Por lo que se constató que a mayor desarrollo vocacional eficaz más influencia ejerció el gusto y la preferencia en las elecciones realizadas.

Por otra parte, señalar que el resto de correlaciones significativas lo hicieron en sentido opuesto. Es decir tuvieron un carácter negativo. El item 31 (la influencia de mi discapacidad) obtuvo la correlación más elevada de las negativas $(r=-.46, p<.05)$, seguido del item 15 (la suerte o la casualidad; $r=-.45, p<.05$ ) y del item 32 (Adaptabilidad del entorno a mi discapacidad; $r=-.42$, $\mathrm{p}<$.05). Los ítems 33 (Recursos materiales y humanos disponibles para mi integración), 16 (el tiempo y el horario de que dispongo) y el 20 (El que sea una carrera/ trabajo que se ajusta a mi condición de hombre o mujer) presentaron correlaciones negativas algo más bajas que las anteriores que oscilaron entre $r=-.38(p<.05)$ y $r=-.36(p<.05)$. Dados estos resultados podemos señalar que a mayor desarrollo vocacional menos influencia tuvieron en la elección vocacional la propia discapacidad, la suerte o casualidad, la adaptabilidad del entorno a su discapacidad, el horario y el tiempo disponible, los recursos materiales para su integración y los estereotipos de género.

Aún no siendo significativas el resto de correlaciones se constató que las correlaciones fueron 
positivas en aquellos ítems que hacen referencia a aspectos personales y por lo tanto relacionados con factores de tipo psicogénico. El resto de correlaciones relacionadas con factores de carácter sociogénico, oportunidad y discapacidad fueron negativas.

TABLA 2. Correlación entre ítems de Factores de elección vocacional y puntuación total de Biodatos

\begin{tabular}{|c|c|}
\hline Items Factores de Elección Vocacional & Biodatos \\
\hline 17. Mis gustos y preferencias &, $55^{* *}$ \\
\hline 31. La influencia de mi discapacidad &,$- 46^{*}$ \\
\hline 15. La suerte o la casualidad &,$- 45^{*}$ \\
\hline 32. Adaptabilidad del entorno a mi discapacidad &,$- 42^{*}$ \\
\hline 33. Recursos materiales y humanos disponibles para mi integración &,$- 38^{*}$ \\
\hline 16. El tiempo y el horario de que dispongo &,$- 37^{*}$ \\
\hline 20. El que sea una carrera/ trabajo que se ajusta a mi condición de hombre o mujer &,$- 36^{*}$ \\
\hline 29. La confianza que tengo en desarrollar con éxito la profesión a la que quiero dedicarme & ,29 \\
\hline 4. Mi preparación personal a lo largo de los años & ,18 \\
\hline 27. Mi constancia, capacidad de trabajo y sacrificio & ,15 \\
\hline 26. El conocimiento que ya tenía sobre el mundo del trabajo al que me dirijo &, 11 \\
\hline 18. El asesoramiento vocacional recibido & 02 \\
\hline 30. El prestigio social de la profesión que quiero desempeñar &,- 03 \\
\hline 12. La confianza que tengo en desarrollar con éxito estos estudios &,- 04 \\
\hline 24. El apoyo, estímulo y ayuda de algún/ a profesor/ a &,- 04 \\
\hline 25. El conocimiento que tengo sobre mí mismo, mis capacidades y mis posibilidades de éxito &,- 05 \\
\hline 19. El haber conocido a una persona importante del área vocacional que he elegido &,- 05 \\
\hline 11. El poder económico y social que pretendo conseguir &,- 08 \\
\hline 28. La facilidad de los estudios y su corta duración &,- 08 \\
\hline 1. La influencia de mi padre (deseos, gustos, éxitos, consejos, profesión,...). &,- 10 \\
\hline 9. La nota de la prueba de selectividad &,- 11 \\
\hline 13. La novedad de los estudios y de las profesiones que implican &,- 15 \\
\hline $\begin{array}{l}\text { 23. El éxito en mis estudios en todas o en algunas materias relacionadas con lo que estoy } \\
\text { haciendo }\end{array}$ &,- 17 \\
\hline 2. El ambiente de casa &,- 17 \\
\hline 6. Las salidas profesionales de lo que he decidido estudiar &,- 17 \\
\hline 5. La dificultad de los estudios y su larga duración &,- 19 \\
\hline 7. Las posibilidades económicas de mi familia (muchas/ pocas) &,- 22 \\
\hline 14. La influencia de algún familiar (deseos, gustos, éxitos, consejos, profesión,...) &,- 23 \\
\hline $\begin{array}{l}\text { 3. Estar "al tanto" de las posibilidades que se han cruzado en mi camino (cursos, viajes, } \\
\text { reuniones, amigos...) }\end{array}$ &,- 24 \\
\hline 10. La influencia de mi madre (deseos, gustos, éxitos, consejos, profesión,...) &,- 26 \\
\hline $\begin{array}{l}\text { 8. Aprovechar las oportunidades de formación que se me han presentado (cursos, } \\
\text { conferencias,...) }\end{array}$ &,- 26 \\
\hline
\end{tabular}




\begin{tabular}{|l|r|}
\hline 21. El prestigio social de los estudios &,- 29 \\
\hline 22. El poder estudiar cerca de mi residencia sin tener que desplazarme &,- 30 \\
\hline 34. Barreras arquitectónicas que pudiesen influir en el desarrollo de mi formación &,- 36 \\
\hline
\end{tabular}

Nota. ${ }^{*} p<.05 \quad{ }^{* *} p<.01$

Además se correlacionó la puntuación de Biodatos (desarrollo vocacional eficaz) con los Factores de elección vocacional. En la tabla 3 se observa, que la única correlación estadísticamente significativa fue la que se obtuvo entre la discapacidad y la puntuación total de biodatos, siendo ésta una correlación moderadamente alta y negativa $(r=-.43, p<.05)$. En este sentido, se verificó que los sujetos con mayor desarrollo vocacional eficaz se ven menos afectados por la influencia de la discapacidad en su elección vocacional. Del mismo modo es de destacar, aún no siendo significativas, las correlaciones negativas que aparecieron, como ya se prevía según los resultados obtenidos en las correlaciones con los ítems, en torno a otros factores de elección como: los condicionantes académicos, la preparación para la oportunidad, estereotipos, oportunidad de formación y condicionamientos familiares.

Por otra parte, la madurez vocacional, como era de esperar, correlacionó de manera positiva, aunque no de manera estadísticamente significativa, con la puntuación de biodatos. De manera que los sujetos con un mayor desarrollo vocacional eficaz son lo que presentaron una mayor madurez vocacional. También conviene precisar la ausencia de relación entre el asesoramiento y los biodatos.

\section{TABLA 3. Correlación entre la puntuación total de Biodatos y los Factores de elección vocacional}

\begin{tabular}{|l|c|}
\hline \multicolumn{1}{|c|}{ Factores de elección vocacional } & Biodatos \\
\hline Condicionantes familiares & -.25 \\
\hline Estereotipos. poder y prestigio & -.28 \\
\hline Condicionantes académicos & -.34 \\
\hline Madurez vocacional & .32 \\
\hline Asesoramiento vocacional & -.03 \\
\hline Preparación para la oportunidad & -.32 \\
\hline Oportunidad de formación & -.27 \\
\hline Discapacidad & $-.43^{*}$ \\
\hline
\end{tabular}

Nota. ${ }^{*} p<0.05$

Análisis de regresión tomando como variable dependiente el desarrollo vocacional y como variables independientes los factores de elección

Con el fin de comprobar que factores de elección vocacional eran los mejores predictores del Desarrollo vocacional eficaz (puntuación total de biodatos) se realizó un análisis de regresión lineal. Se obtuvo un modelo de regresión significativo $\left[F(30,8)=2.881, R=.74, R^{2}=.55, p<.05\right]$ que explicó un $55 \%$ de la varianza de los Biodatos, siendo los mejores predictores del desarrollo vocacional eficaz la madurez vocacional en sentido positivo y la discapacidad en sentido negativo. Es decir el tener una alta influencia de la madurez vocacional y una baja influencia de la 
discapacidad en la elección vocacional predice tener un desarrollo vocacional eficaz. Aparece por tanto el papel de factores psicógenicos y la propia discapacidad como los mejores predictores del desarrollo vocacional.

TABLA 4. Análisis de regresión lineal: biodatos (VD), factores de elección (VI)

\begin{tabular}{|c|c|c|c|c|c|}
\hline Predictores & B & Error típico & Beta & t & Sig \\
\hline Madurez vocacional & 2.953 & 1.049 & .569 & 2.815 & * \\
\hline Discapacidad & -1.017 & .411 & -.425 & -2.472 & * \\
\hline
\end{tabular}

Nota. ${ }^{*} p<.05$

\section{Análisis inferenciales}

Para comprobar si existían diferencias entre los grupos según el desarrollo vocacional eficaz (bajo, medio y alto), fueron empleadas pruebas no paramétricas para muestras independientes. En este caso tras aplicar la prueba de Kruskal-Wallis, fueron encontradas diferencias significativas tan solo en el factor discapacidad $\left(X^{2}(2,30)=7.177, p<.05\right)$.

Realizada la prueba $U$ de Mann-Whitne para verificar donde se localizaban esas diferencias, se obtuvo que las diferencias se dieron entre los grupos alto y bajo ( $U$ de Mann-Whitne $=14.000$, $\mathrm{p}<.05$ ) y alto y medio ( $U$ de Mann-Whitne $=33.5, \mathrm{p}<.05$ ), no encontrándose diferencias entre el bajo y el medio. Se ratifica que cuanto mayor es el desarrollo vocacional menos influencia tuvo la discapacidad en la elección vocacional.

Con respecto al resto de factores, aunque no se obtuvieron diferencias significativas, se constató que conforme se incrementa el desarrollo vocacional las puntaciones de los factores condicionantes familiares, estereotipos de género, condicionantes académicos sufren un descenso paulatino conforme se incrementa el desarrollo vocacional. Hecho que contrasta con la madurez vocacional que incrementa sus puntaciones conforme el desarrollo vocacional es más alto.

TABLA 5. Análisis diferencial de los factores de elección vocacional en relación el tipo de desarrollo vocacional

\begin{tabular}{|c|c|c|c|c|c|}
\hline \multirow{2}{*}{ Factores } & \multicolumn{2}{|c|}{ Desarrollo Vocacional (Biodatos) } & Media (Sd) & Chi-Cuadrado $\left(x^{2}\right)$ & Sig. \\
\cline { 2 - 6 } & $\begin{array}{c}\text { Bajo } \\
\mathbf{n = 7}\end{array}$ & $\begin{array}{c}\text { Medio } \\
\mathbf{n = 1 1}\end{array}$ & $\begin{array}{c}\text { Alto } \\
\mathbf{n}=12\end{array}$ & 3.442 & .179 \\
\hline $\begin{array}{c}\text { Condicionantes } \\
\text { familiares }\end{array}$ & $2.69(1.13)$ & $2.47(1.11)$ & $1.82(0.74)$ & 5.118 & .077 \\
\hline $\begin{array}{c}\text { Estereotipos, poder y } \\
\text { prestigio }\end{array}$ & $2.71(0.93)$ & $2.56(0.71)$ & $1.94(0.63)$ & 4.297 & .117 \\
\hline $\begin{array}{c}\text { Condicionantes } \\
\text { académicos }\end{array}$ & $2.97(0.63)$ & $2.64(0.86)$ & $2.18(0.66)$ & 1.891 & .388 \\
\hline Madurez vocacional & $2.91(0.93)$ & $2.90(0.50)$ & $3.28(0.71)$ & & \\
\hline
\end{tabular}




\begin{tabular}{|c|c|c|c|c|c|}
\hline $\begin{array}{c}\text { Asesoramiento } \\
\text { vocacional }\end{array}$ & $2.19(1.43)$ & $2.63(1.11)$ & $2.11(0.80)$ & 1.517 & .468 \\
\hline $\begin{array}{c}\text { Preparación para la } \\
\text { oportunidad }\end{array}$ & $3.06(0.44)$ & $3.10(0.63)$ & $2.64(0.62)$ & 4.334 & .114 \\
\hline $\begin{array}{c}\text { Oportunidad de } \\
\text { formación }\end{array}$ & $3.00(0.60)$ & $3.06(0.70)$ & $2.44(0.59)$ & 5.305 & .070 \\
\hline Discapacidad & $3.32(1.07)$ & $2.91(1.46)$ & $1.90(1.07)$ & $7.177^{\star}$ & .028 \\
\hline
\end{tabular}

Nota. ${ }^{*} \mathrm{p}<.05$

\section{Conclusiones}

El objeto del presente estudio fue comprobar la relación existente entre el desarrollo vocacional y los factores de elección vocacional en titulados universitarios con discapacidad insertados laboralmente. Como indican Vila y Pallisera (2002) y Coleman (2008) este tipo de población apenas ha sido estudiada tanto a nivel nacional como internacional. Desde este punto de vista, la novedad de este estudio reside en el tipo de sujetos que constituyó la muestra seleccionada. Creemos que el conocimiento del desarrollo vocacional de estos sujetos puede repercutir de forma positiva en el asesoramiento vocacional y profesional de este colectivo.

De manera similar Rodriguez, Suso, Vázquez y Velasco (2013) en un estudio sobre discapacidad, estudio superior y mercado de trabajo, encontraron que las personas con discapacidad se decantaban a priori, en mayor medida por motivaciones vocacionales que por razones estrictamente prácticas, como la elección de los estudios con mayores salidas laborales. Como contrapunto, destaca la poca influencia que ejerció su discapacidad a la hora de tomar esta decisión. Otro estudio realizado por Dalmau, Llinares y Giné (2010) apoya nuestros resultados, demostrando que solo un $29.87 \%$ de los estudios universitarios escogidos estuvieron influenciados por su discapacidad, el resto de los participantes opinó que no les influyó para nada en su elección. Castignani (2011) en un estudio realizado con discapacitados visuales demuestra que los factores que priorizan en su elección, están relacionados con el interés por las tareas que se desarrollan en la profesión.

Concretando nuestro concepto de desarrollo vocacional eficaz como la manifestación de un claro interés y satisfacción por la opción elegida, mantenida en el tiempo y adecuada a sus aptitudes y visión de futuro profesional, podemos decir que más de la mitad de la muestra tendría este perfil vocacional. Constatamos este hecho a través de la influencia que ejerció la discapacidad en la elección de la carrera de los participantes, ya que más de la mitad de ellos confirmó que no les influyó para nada en su elección. Resultados similares se obtienen en otros estudios (Castignani, 2011; Dalmau, Llinares y Giné, 2010 y Rodriguez, Suso, Vázquez y Velasco, 2013).

En cuanto a los factores de elección vocacional, se constató que son los de tipo psicogénico los que dominan e influyen en su conducta vocacional, en concreto sus gustos y preferencias son los que ejercen mayor influencia en la toma de decisiones preprofesionales, seguido por su preparación personal y conocimiento que tenían de sí mismos. Estos resultados están consonancia con otros estudios (Martinez-Vicente, Segura y García, 2014).

Por otra parte surge la oportunidad (Pascual, 2006) tanto en su preparación como en su formación, como un factor relevante en personas con discapacidad. Esto puede ser debido a que dadas sus circunstancias se encuentran más alerta, con el fin de no dejar escapar ocasiones que se les pueden presentar como algo beneficioso para el desarrollo de su carrera o inserción laboral. 
No se observa a su vez, ninguna relación significativa entre desarrollo vocacional eficaz, con factores de carácter sociogénico, como pueden ser los condicionantes familiares, el asesoramiento recibido o los estereotipos poder/prestigio. Sin embargo, algunas investigaciones realizadas con anterioridad señalan los condicionantes familiares (Valls, Vilà y Pallisera, 2004; Wagner, Newman, Cameto y Levine i Marder, 2007) como muy influyentes en el desarrollo académico y profesional de personas con discapacidad destacando como factor muy influyente la sobreprotección recibida.

En cuanto al asesoramiento recibido por parte de las instituciones educativas, se percibió que la mayoría de la muestra no recibió o fue ineficaz, ya que no tuvo ningún tipo de repercusión en su elección vocacional. El estudio llevado a cabo por Campo, Verdugo, Díez y Sancho (2006), confirma este hecho, ya que reveló una repercusión heterogénea y desigual de los servicios de apoyo y asesoramiento destinados a este tipo de población. Por su parte, Peralta (2007), resalta los avances que se han realizado en el asesoramiento universitario de discapacitados, aunque todavía quedan lejos de asegurar los principios de igualdad e inclusión establecidos a nivel europeo.

Encontramos por tanto, que los sujetos con discapacidad no se limitan por factores externos en su desarrollo vocacional, sino que es la propia sociedad la que puede limitar o poner barreras.

Los análisis de asociación entre Biodatos (Desarrollo Vocacional) y Factores de elección nos permiten concluir que los sujetos con mayor desarrollo vocacional eficaz se ven menos afectados por su discapacidad. Por otra parte, se verificó que los sujetos que contaban con un desarrollo vocacional eficaz son los que presentaron mayor madurez vocacional. Estos resultados se ratifican en el modelo de regresión realizado que atribuye el mayor peso predictivo del desarrollo vocacional a ambas variables. Además señalar que el factor discapacidad es el único que marcó diferencias según el tipo de desarrollo vocacional, en el sentido de a mayor desarrollo vocacional eficaz menor es su influencia en la elección vocacional. Así mismo, algunos estudios realizados con titulados universitarios discapacitados (Gill 2007; Webster, 2004) afirman que este tipo de población presenta características propias como: la capacidad de perseverar en sus logros, tener alta autoestima, enfocarse en acciones posibles, no frustrarse ante fracasos, etc. Lo que confirma que la discapacidad no juega un papel de limitación, si no de motivación para su propia trayectoria personal y profesional con la intención de poner a prueba su valía y eliminar de su entorno más próximo los diferentes estereotipos o etiquetas que han ido asumiendo a lo largo de su trayectoria escolar y formativa.

Las conclusiones generales que podemos extraer de nuestro trabajo muestran que el tipo de población estudiada es consciente y conocedora de sus posibilidades, por eso su discapacidad junto a otros factores de carácter sociogénico se muestran como no influyentes. Se constata que han construido su propio desarrollo vocacional teniendo presente sus limitaciones. Presentan además características de madurez vocacional como son: expectativas de éxito en los estudios, gustos y preferencias, autoconocimiento, conocimiento del mundo laboral, constancia, capacidad de trabajo y sacrificio, este dato se ha mostrado como determinante en esta muestra para la consecución de un desarrollo vocacional eficaz. Por lo tanto, y conociendo estos datos, sería de especial importancia tener en cuenta estos aspectos en el asesoramiento vocacional en este tipo de colectivo para favorecer su desarrollo vocacional y facilitar una transición académica y sociolaboral satisfactoria.

En cuanto a las limitaciones del estudio vienen dadas sobre todo por el número de sujetos que constituye la muestra estudiada así como por las características de los propios instrumentos empleados que son medidas de autoinforme. Por otra parte, no se ha tenido en cuenta como variable el tipo de discapacidad dada la reducida muestra. Destacar el carácter preliminar y exploratorio de este estudio cuya pretensión es ser el punto de partida para profundizar en el conocimiento de las características vocacionales de este colectivo ya que han sido escasamente 
estudiadas. Desde nuestro punto de vista el valor que presenta este estudio, se centra en el tipo de muestra seleccionada, ya que representa dentro del colectivo de la discapacidad aquellos sujetos que han tenido éxito en la consecución y mantenimiento de un puesto de trabajo. Futuras investigaciones deberían centrarse en incrementar el número de sujetos de la muestra lo que permitiría obtener resultados relacionados con otras variables como el tipo de discapacidad, la ocupación desempeñada, el papel del género etc. Esto nos permitiría incrementar el conocimiento del papel que tienen los factores de elección en el desarrollo vocacional eficaz pudiendo identificar los que quedan asociados a un desarrollo vocacional eficaz. Esto facilitaría el desarrollo de intervenciones que llevaran a estimular y mejorar el desarrollo vocacional de las personas con discapacidad. Además surge otra línea de investigación en la que se tengan en cuenta otras variables asociadas a las tratadas en este estudio. En este sentido sería oportuno comprobar la relación de estas con variables con otras tales como: las características personales, la preparación para la carrera profesional, el proceso de transición al mundo laboral y la satisfacción con el trabajo desempeñado.

\section{Referencias bibliográficas}

Amir, T., Gati, I. y Kleiman, T. (2008). Understanding and interpreting career decision-making difficulties. Journal of Career Assessment, 16, 281-309. Doi: 10.1177/1069072708317367.

Ávila, V. y Rocabert, E. (2001). Características vocacionales de estudiantes universitarios ciegos y deficientes visuales: una aproximación cualitativa. Revista Española de Orientación y Psicopedagogía, 22, 319-340.

Ávila, V, Celeste, M. y Rocabert, E. (2003). Discapacidad y asesoramiento vocacional. En F. Rivas (Ed.), Asesoramiento vocacional. Teoría, Práctica y asesoramiento vocacional (pp. 465-493). Barcelona: Ariel.

Cabrera, L. y Bethencourt, J. T. (2008). Desarrollo vocacional y estrategias de aprendizaje en estudiantado universitario. Revista de Orientación Educacional, 22, 13-29.

Coleman W. B. (2008). Transition from college to work: Lived employment experiences and perceptions of college seniors and recent college graduates with physical disabilities seeking employment opportunities. (Unpublished )Dalmau, M., Llinares, M., Sala, I., y Giné, C. (2010). Integración laboral de los universitarios españoles con discapacidad. Fundación Universia. Barcelona.

Chethan, G., Smithh, S., Elliot, W. y Friedline, T. (2013). Family assets, postsecondary education and students with disabilities: building on progress and avercoming challenges. Children and Yoth Servces Review, 35 (7), 1078-1086.

Dosil, A. y Fernandez Eire,L.F. (2001). O Self- Directed Search (SDS) en Galicia. Revista Gallega do Encino, 33, 137-153.

Gati, I., Landman, S., Davidovitch, S., Asulin-Peretz, L. y Gadassi, R. (2010). From career decision-making styles to career decision-making profiles: A multidimensional approach. Journal of Vocational Behavior, 76, 277-291. Doi: 10.1016/j.jvb.2009.11.001.

Gill, L. A. (2007) Bridging the transition gap from high school to college: Preparing students with 
disabilities for a successful postsecondary experience. Teaching Exceptional Children, 40 (2), 12-15.

Gimeno, M.J. (2003). Análisis de la problemática vocacional y de los biodatos ante la Toma de Decisiones. Tesis Doctoral. Universidad de Valencia.

González Maura, V. (2006). La formación de competencias profesionales en la universidad. Reflexiones y experiencias desde una perspectiva educativa, en XXI. Revista de Educación, $8,175-188$.

Gomez-Artiaga, T., Lloret, S., Rocabert, E., y Descals, A. (2013). Desarrollo y validación del Cuestionario de Personalidad Vocacional Adaptativa: un cuestionario para analizar la conducta vocacional de estudiantes universitarios. Anales de psicología, 2, 470-481.

Luque, D. J. (2006). Orientación Educativa e Intervención Psicopedagógica en el alumnado con discapacidad. Análisis de casos prácticos. Málaga. Aljibe.

Martín, T. (2006). Orientación vocacional. Luventicus (Academia de ciencias) Rosario. Argentina.

Martín del Buey, F., Martín, E., Fernández, A., Dapelo, B. y Marcone, R. (2004). Evaluación de la personalidad eficaz en contextos educativos: primeros resultados. Revista de Orientación Educacional, 33-34, 79-101.

Martínez-Vicente, J.M., Segura, M. A. y García, I. (2014). Desarrollo vocacional y factores que influyeron en la elección vocacional en personas discapacitadas con estudios universitarios insertadas laboralmente. International Journal of Development and Educational Psychology, 3, 539-548.

Martínez-Vicente, J. M. y Rocabert, E. (2014). Desarrollo vocacional y preparación de la carrera profesional en estudiantes universitarios. International Journal of Development and educational Psychology,5, 405-416.

Mehran, F. (2010). Psychologie positive et personnalité. Paris: Elsevier-Masson SAS.

Mora, R. (2008). Conducta y asesoramiento vocacional/preprofesional de los estudiantes universitarios: Variables individuales del desarrollo de la carrera y servicios institucionales. Tesis doctoral. Universidad de Valencia.

Pascual, F. (2006). Conducta e identidad vocacional en la adolescencia: una aproximación empírica. Tesis doctoral. Valencia. Universidad (Estudi General) de Valencia, Facultad de Psicología.

Peralta, A. (2007). Libro Blanco sobre Universidad y Discapacidad. Madrid: Real Patronato sobre Discapacidad.

Polo, M.T., y López-Justicia, M.D.(2006). Actitudes hacia las personas discapacidad de estudiantes de la Universidad de Granada. Revista Española de Orientación y Psicopedagogía, 17,195 - 211.

Rivas, F. (1988). Psicología Vocacional. Enfoques del asesoramiento. Madrid: Morata.

Rivas, F. (2003). Asesoramiento vocacional. Teoría, práctica e instrumentación. Barcelona: Ariel Psicología.

Rivas, F. (2005). Conducta Vocacional y Asesoramiento Profesional de los Estudiantes Universitarios: Protocolo Informático para la Autoayuda Preprofesional. Informe proyecto I+D 
BS02001-3150. Valencia.

Rivas, F. y Pascual, F. (2003). Inventario de factores vocacionales. En F. Rivas, Asesoramiento vocacional. Teoría, práctica e instrumentación. Barcelona: Ariel

Rocabert, E., Descals, A. y Gómez, A. (2007). Los Biodatos como indicadores de la conducta vocacional universitaria: Elaboración de un instrumento de evaluación. Electronic Journal of Research in Educational Psychology, 5, 15-36.

Rocabert, E y Martínez-Vicente, J.M. (2011). Conducta vocacional y preprofesional de los estudiantes universitarios. Almería: Education \& Psychology I+D+i.

Staudinger U. M., Bowen, C. E. (2010). Life-span perspectives on positive personality development in adulthood and old age. In M. E. Lamb, A. M. Freund, y R.M. Lerner (Eds.), The handbook of life-span development, vol. 2: Social and emotional development (pp. 254-297). Hoboken, NJ, US: John Wiley \& Sons Inc.

Urribarri, L. (2004). Programa Decidiendo mi profesión, propuesta de orientación profesional para la educación media. Maracaibo: Ed. Venezuela.

Valiante, G. (2000). Writing Self-efficacy and Gender Orientation. A developmental Perspective. A Dissertation Proposal. Atlanta: Emory University.

Valls, M., Vilà, M. y Pallisera, M. (2004). La inserción de las personas con discapacidad en el trabajo ordinario. El papel de la familia. Revista de Educación, 334, 66.

Vila, M., y Pallisera, M. (2002). La integración socio laboral de personas con discapacidad y formación superior. Revista Educación Especial, 33, 51-71.

Wagner, M., Newman, L., Cameto, R. y Levine i Marder, C. (2007). Perceptions and expectations of youth with disabilities. A special topic report of findings from the national longitudinal transition study-2 (NLTS2). No. (NCSER 2007-3006).U.S. Department of Education.

Webster, D. D. (2004). Giving Voice to Students with Disabilities Who Have Successfully Transitioned to College. Career Development for Exceptional Individuals, 27, 151-180. Doi: $10.1177 / 088572880402700203$.

White, G., Summers, J. A., Zhang, E. y Renault, V. (2014). Evaluating the effects of a a SelfAdvocacy Training Program for Undergraduates with Disabilities. Journal of Postsecondary Education and Disability, 27 (3), 229-244.

\section{Fuentes electrónicas}

Campo, M., Verdugo, M.A., Díez, E. y Sancho, I. (2006). Mirando al futuro: Comparación crítica de la situación de los servicios españoles de atención a los universitarios con discapacidad. En Adaptar la Igualdad, Normalizar la Diversidad: II Congreso Nacional sobre Universidad y Discapacidad. XI Reunión del Real Patronato sobre Discapacidad. Madrid: Universidad Complutense de Madrid. Recuperado de http://www.fedaes.org/bol/bol51/l1\%20Congreso\%20Universidad-Discapacidad.pdf

Castignani, M. L. (2011). La orientación vocacional en alumnos con discapacidad visual. 3은 Congreso Internacional de Investigación. Recuperado de 
http://www.memoria.fahce.unlp.edu.ar/trab eventos/ev.1514/ev.1514.pdf.

Dalmau, M., Llinares, M., Sala, I. y Giné, C. (2010). Integración laboral de los universitarios españoles con discapacidad. Detección de las fortalezas y debilidades en el momento del acceso al mercado laboral español. Percepción de los universitarios y percepción de las empresas. Extraído el 16 de junio de 2013, de: http://fundacionuniversia.net/programas/informacion/informes/detalleProgramas-1570.html

Rodriguez, P., Suso, A., Vázquez, D. y Velasco, G. (2013). Discapacidad, estudios superiores y mercado de trabajo. Barreras de acceso y repercusión en la inserción laboral. Fundación ONCE. Recuperado

http://www.fundaciononce.es/ES/Publicaciones/estudios/Documents/Informe FINAL Estudios Superiores.pdf.

Rodríguez-Martín, Alejandro, \& Álvarez-Arregui, Emilio. (2015). Universidad y discapacidad: Actitudes del profesorado y de estudiantes. Perfiles educativos, 37(147), 86-102. Recuperado en 01 de octubre de http://www.scielo.org.mx/scielo.php?script=sci arttext\&pid=S018526982015000100006\&lng=e $\underline{s \& t \operatorname{lng}=e s}$

Fecha de entrada: 18 de febrero de 2015

Fecha de revisión: 14 de mayo de 2015

Fecha de aceptación: 19 de octubre de 2015 